\title{
Die inhaltsanalytische Bilanz einer Dekade
}

\section{Autor_innen, 177 empirische Beiträge - 10 Jahre Frühe Bildung}

\author{
Felix Berth¹ und Hanna Schneider ${ }^{2}$ \\ 'Abteilung Kinder und Kinderbetreuung, Deutsches Jugendinstitut, München \\ ${ }^{2}$ Pädagogische Hochschule Weingarten / Deutsches Jugendinstitut
}

\begin{abstract}
Zusammenfassung: Der Beitrag betrachtet die Entwicklung der Zeitschrift Frühe Bildung im ersten Jahrzehnt ihres Erscheinens. Mit einem inhaltsanalytischen Instrumentarium wird nachgezeichnet, was die Autorenschaft der Zeitschrift kennzeichnete, welche Methoden in den Beiträgen verwendet und welche thematischen Schwerpunkte gesetzt wurden. Die Analyse des Korpus, der 177 Artikel aus den Jahren 2011 bis 2020 umfasst, weist darauf hin, dass die anfänglich dominierende psychologische Ausrichtung auf Seiten der Autor_innen durch eine stärkere Präsenz von pädagogischen Wissenschaftler_innen abgelöst wurde. Damit ging eine Zunahme qualitativer Forschungsdesigns einher; dennoch blieben quantitative Methoden in der Frühen Bildung vorherrschend. Thematisch standen der kindliche Kompetenzerwerb sowie die Praxis der Kindertageseinrichtungen im Vordergrund; deutlich seltener wurden Arbeiten publiziert, die sich mit Ausbildungsfragen oder eigenständigen Kinderperspektiven befassten.
\end{abstract}

Schlüsselwörter: Frühe Bildung, Zeitschriftenanalyse, qualitative Inhaltsanalyse

Content Analysis of a Decade's Work: 511 Authors, 177 Contributions - 10 Years of Frühe Bildung

Abstract: This paper analyzes the development of the journal Frühe Bildung [Early Education] in the first decade of its publication. Using content analysis, we trace the authorship of the contributions, the methods applied in the articles, and their thematic focus. With this corpus, comprising 177 articles from 2011 to 2020, we find that the initially dominant psychological orientation on the part of the authors was replaced by a stronger presence of pedagogical experts, accompanied by an increase in qualitative research designs. Nevertheless, quantitative methods remained more prevalent than qualitative ones over the years. Thematically, children's competencies as well as the practices of daycare centers remained in the foreground. Significantly fewer papers dealt with staff training or children's perspectives.

Keywords: early childhood education, journal analysis, content analysis

Wissenschaftliche Zeitschriften boomen. Nimmt man beispielsweise mit dem Scimago Journal \& Country Rank ${ }^{1}$ eine grobe Zählung vor, stellt man fest, dass es im Jahr 2000 weltweit 503 Zeitschriften der Disziplin social sciences / education und 788 Zeitschriften in der Disziplin psychology gab. Knapp zwei Jahrzehnte später, im Jahr 2019, waren es dann mit 1401 in den Erziehungswissenschaften und 1303 in der Psychologie ungefähr doppelt so viele. Die Genauigkeit dieser Zahlen kann man bezweifeln, schließlich fehlen in der Datenbank gewiss etliche Publikationen, und auch manches Pseudo-Journal, dessen Geschäftsmodell allein im Einstreichen von open-access-Gebühren besteht, dürfte enthalten sein. Dennoch ist der Trend eindeutig: Die Zahl der akademischen Zeitschriften wächst schnell, und ihre Bedeutung im Wissenschaftssystem nimmt zu.

Diese Entwicklung gilt einerseits für Disziplinen wie die Medizin und die Psychologie, in denen der wissenschaftliche Fortschritt seit langem in Zeitschriften zur Diskussion gestellt wird (Fleck, 1936/1983). Andererseits erfasst der Trend auch die Philosophie oder die Erziehungswissenschaften, in denen Monografien lange Zeit größere Bedeutung hatten als Aufsätze (Bray \& Major, 2011).

Die Frühe Bildung, die wir im Folgenden genauer betrachten wollen, ist aus dieser globalen Perspektive ein kleines Puzzlestück in einem sehr großen Bild: Im Jahr 2011 zum ersten Mal erschienen, ist sie eine unter Hun- 
derten von neuen Zeitschriften in den Feldern von Psychologie und Pädagogik. Aus einer nationalen Sicht stellt sich die Situation allerdings etwas anders dar. Hier war die Frühe Bildung - soweit wir sehen - die einzige ZeitschriftenNeugründung, die auf den deutschen Boom der frühpädagogischen Forschung seit der Jahrtausendwende folgte. Mit ihrem Anspruch der Interdisziplinarität begab sie sich auf schwieriges Terrain; mit ihrer peer-review-Vorgabe formulierte sie einen markanten Qualitätsanspruch. Nach knapp zehn Jahren, in denen 37 Hefte mit insgesamt 386 Beiträgen publiziert wurden, erscheint uns eine Zwischenbilanz angebracht. Den weltweiten Bedeutungszuwachs wissenschaftlicher Zeitschriften können wir damit zwar nicht nachzeichnen, eine für Deutschland relevante Entwicklung aber durchaus.

Unser Versuch konzentriert sich dabei nicht auf den Erkenntnisfortschritt, zu dem die Frühe Bildung beitrug, sondern auf die Modalitäten der Wissensproduktion, die man am Endprodukt ablesen kann: Was charakterisiert diese Zeitschrift, welche Autor_innen schreiben darin? Welche Methoden verwenden die Beiträge, welche Themen sind die wichtigsten? Damit steht die Arbeitsweise dieser Publikation im Fokus; unser Blick ist mindestens so stark wissenssoziologisch wie pädagogisch/psychologisch-bilanzierend geprägt. Wenn es auf diese Weise gelingt, das Profil der Frühen Bildung herauszuarbeiten, ist auch ein kleines Stück (Inter-)Disziplingeschichte geschrieben.

\section{Das methodische Vorgehen}

Um die Charakteristika der Zeitschrift zu beschreiben und ihre Entwicklung nachzuzeichnen, haben wir ein inhalts- analytisches Verfahren gewählt. Beginnend mit der Nullnummer vom Herbst 2011 und endend mit der vierten Ausgabe im Jahr 2020, bildeten wir einen Korpus aus den 177 Artikeln der Rubriken Schwerpunktbeiträge und Freie Beiträge. Diese Beiträge basieren - so der Anspruch der Zeitschrift - auf eigenständigen empirischen Forschungen und stellen damit den wissenschaftlichen Kern der Frühen Bildung dar. Ausgeblendet werden damit die Beiträge der übrigen Rubriken Rezensionen, Diskussionen, Innovationen sowie Informationen (vgl. Abb. 1), weil sie in den allermeisten Fällen nicht das Ziel haben, eigenständige Forschungen mit Ergebnissen darzustellen, sondern allgemeiner über das frühpädagogische Feld informieren, einzelne Projekte eher nachrichtlich präsentieren oder Position in Debatten beziehen. Der Fokus unserer Inhaltsanalyse liegt damit auf dem, was die Frühe Bildung zur Erforschung der Kindheit beiträgt.

Die Kategorienbildung erfolgte überwiegend deduktiv, in Abhängigkeit von Theorien und Vorwissen. Wir knüpften dabei insbesondere an Versuche aus den Erziehungswissenschaften an, Publikationen aus dem eigenen Forschungsfeld inhaltsanalytisch (Tenorth, 1986) und historisierend (Kauder \& Vogel, 2015) zu betrachten. Vom groß angelegten Projekt von Zierer und Kollegen (u. a. Zierer, Ertl, Philipps \& Tippelt, 2013) übernahmen wir die drei zentralen Fragen, von denen sich die ersten beiden eher einfach beantworten lassen, die dritte etwas komplizierter ist:

- Autor_innenanalyse: Wer verfasst die Beiträge?

- Methodenanalyse: Welche Forschungsmethoden werden angewandt?

- Themenanalyse: Mit welchen Inhalten befassen sich die Beiträge?

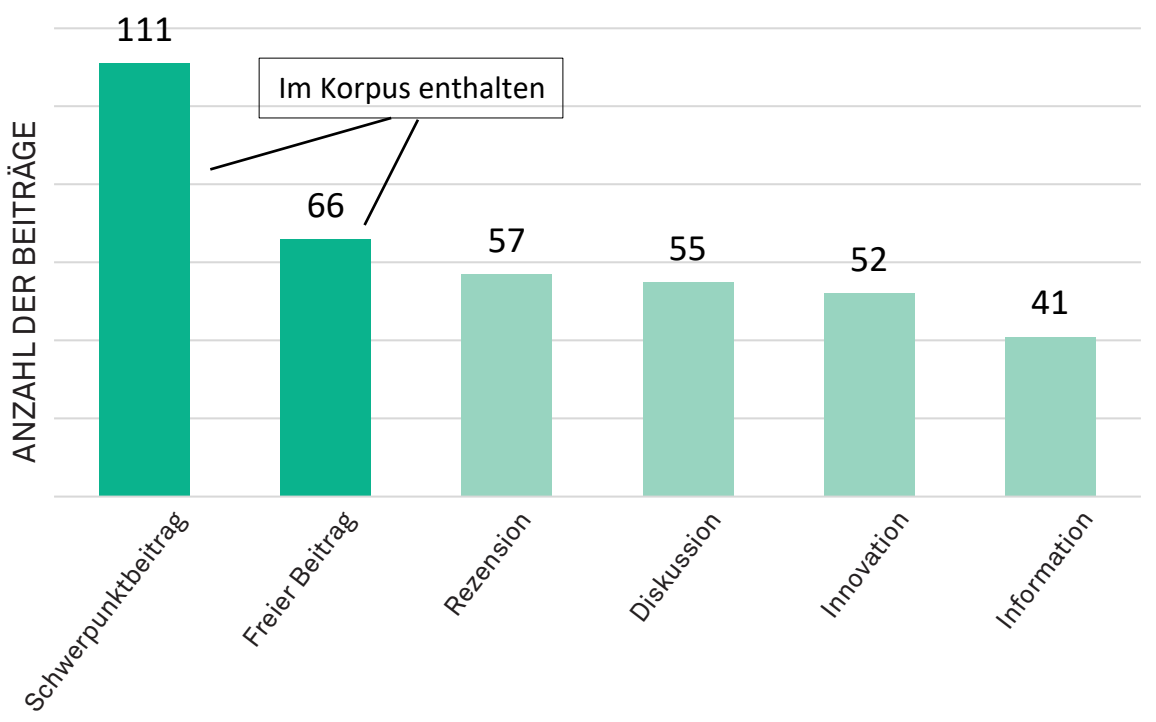

Abbildung 1. Beiträge nach Rubriken und Aufnahme in den Korpus, absolut.

\section{Autor_innenanalyse}

Anhand der in der Zeitschrift genannten Informationen haben wir eine Einteilung der Autor_innen in den Kategorien Geschlecht, Anzahl (Einzel- / Mehrfachautor_innenschaft), Dienstort (Kategorisierung nach Staaten) und institutionelle Anbindung (Universitäten und Hochschulen / Forschungszentren/Sonstige) vorgenommen. Die disziplinäre Zugehörigkeit der Autor_innen (Pädagogik, Psychologie, Soziologie, Sonstige) ermittelten wir durch Internetrecherchen, wobei die im Lebenslauf dominierende Disziplin die Zuordnung bestimmte. War keine 
dominierende Fachrichtung erkennbar, erfolgte die Zuordnung anhand der zuerst genannten Disziplin.

\section{Methodenanalyse}

Die Vorgehensweisen der Beiträge wurden durch eine Analyse der Abstracts sowie ihrer Methodenteile ermittelt. Unterteilt wurden sie in quantitative Methoden, qualitative Methoden und Mixed-Methods-Ansätze. Sekundär- bzw. Metaanalysen wurden der vierten Kategorie systematische Übersichtsarbeiten zugeordnet.

\section{Themenanalyse}

Das Spektrum der Zeitschrift umfasst, so schrieben die Herausgeber_innen im Editorial der ersten Ausgabe, „Fragen der Professionalisierung von pädagogischen Fachkräften, der organisatorischen Verankerung früher Bildung sowie der individuellen Entwicklung von Kindern in frühen Bildungskontexten" (Becker-Stoll et al., 2011, S.1). Daraus entwickelten wir die Oberkategorien Kinder, Personal und Organisation. Weil wir annahmen, dass auch Mütter und Väter in der frühen Bildung von Bedeutung sind und in den Beiträgen thematisiert werden, wurden Eltern als weitere Oberkategorie aufgenommen. Zwei dieser vier Oberkategorien haben wir weiter differenziert, um feinere thematische Analysen zu ermöglichen.

So wurde die Kategorie Kinder unterteilt in kindliche Sichtweisen (Perspektiven von Kindern zu unterschiedlichen Themen, bspw. mit Fokus auf Partizipation in der Kindertageseinrichtung), personale und soziale Charakteristika von Kindern (z.B. Geschlecht, Migrationshintergrund oder Behinderung der Kinder) und kindlicher Kompetenzerwerb (Entwicklung verschiedener kindlicher Fähigkeiten). Die Kategorie Personal wurde gegliedert in die Unterkategorien Ausbildungsort Fachschule sowie Ausbildungsort Hochschule, womit die beiden wichtigsten Ausbildungssettings erfasst werden. Für Beiträge, die mehrere dieser Settings untersuchten, wurde die Unterkategorie Vergleich Ausbildungsorte entwickelt. Die AusbildungsPraxis-Relation wurde für Beiträge vorgesehen, die z.B. Studierende mit bereits in der Praxis tätigem Personal verglichen. Beiträge, die ausschließlich das praktische Tätigkeitsfeld betrachteten, fallen in die Unterkategorie Praxis (Fachkraft-Orientierungen, Prozessaspekte).

Die Kategorisierung erfolgte anhand einer mehrfachen Durchsicht der Beiträge, wobei Titel und Abstract als erste Indizien genutzt wurden. Eine tiefergehende Analyse, bei der die Formulierung der Forschungsfrage(n) im Zentrum des Interesses stand, diente der endgültigen Zuordnung. Beiträge, die zwei Aspekte behandelten - etwa in einer Un- tersuchung von Ursache-Wirkungs-Zusammenhängen -, haben wir nach der wichtigsten unabhängigen Variable kodiert, um die Ursache von Effekten zu berücksichtigen, nicht die Effekte selbst. Bei Designs, in denen drei oder noch mehr relevante Aspekte erkennbar waren - beispielsweise bei Evaluationen von Förder- bzw. Trainingsprogrammen, diagnostischen Verfahren und Beobachtungsinstrumenten - ordneten wir die Beiträge nach dem jeweiligen Ziel bzw. der Zielgruppe der Evaluation respektive des Programms zu.

\section{Die Ergebnisse}

\section{Autor_innenanalyse}

Die 177 Beiträge aus den Rubriken Schwerpunktbeiträge und Freie Beiträge wurden von insgesamt 511 Autor_innen verfasst. Ein beachtlicher Teil der Beiträge, 81 Prozent, entstand in Mehrfachautorenschaft; alleine schreibende und einreichende Autor_innen waren damit relativ selten (vgl. Abb. 2). Damit bestätigt sich auch in der Frühen Bildung ein Trend, den Zierer et al. (2013) für die Erziehungswissenschaften beschrieben haben: Der akademische Einzelautor ist inzwischen in der Minderheit; wenn man eine gängige Metapher strapazieren will, kann man behaupten, dass der Elfenbeinturm vom Großraumbüro ersetzt wurde.

Weil im Berufsfeld der frühen Bildung, meist den Kindertageseinrichtungen, in Deutschland weit über 90 Prozent der Beschäftigten weiblich sind (Autorengruppe Fachkräftebarometer, 2019), stellt sich die Frage, ob sich dieses Geschlechterverhältnis auch in der Erforschung dieses Feldes spiegelt. Ältere Arbeiten konnten dies tendenziell bestätigen. So findet sich bei Martini und Dees (2013) das Ergebnis, dass knapp drei Viertel aller frühpädagogischen Promotionen und Habilitationen von Frauen verfasst wurden, während es in der Bildungsforschung insgesamt nur etwa die Hälfte waren. Je jünger der pädagogisch-psychologische Forschungsgegenstand, umso weib-
Kooperationsform

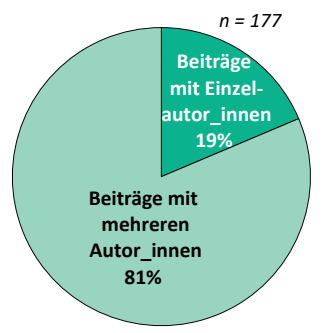

Geschlecht

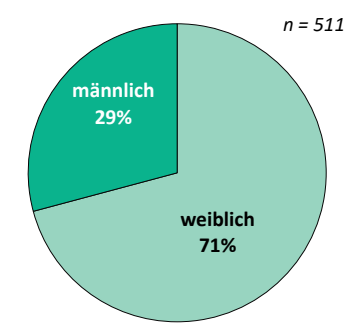

Abbildung 2. Beiträge nach Kooperationsform,

prozentual Beiträge nach Geschlecht der Autor_innen, prozentual. 


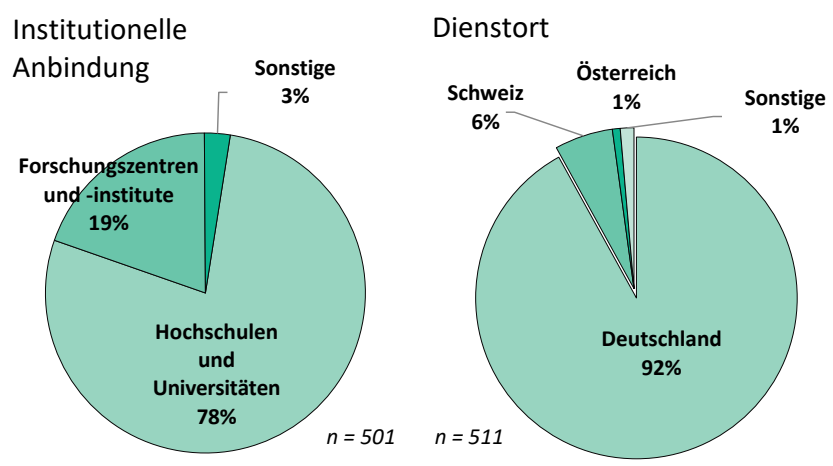

Abbildung 3. Autor_innen nach institutioneller Anbindung, prozentual Autor_innen nach Dienstort, prozentual.

licher die Forschenden, könnte man sagen. Bei den Autor_innenschaften in der Frühen Bildung zeigte sich derselbe Zusammenhang: Der Frauenanteil lag hier im beobachteten Jahrzehnt bei 71 Prozent (vgl. Abb. 2).

Dieser Befund lässt sich als Feminisierung dieser Forschung verstehen, die allerdings schwächer ausfällt als im entsprechenden Praxisfeld. Care-Berufe, so könnte man anknüpfend an dreißig Jahre alte, immer noch gültige Befunde (Rabe-Kleberg, Krüger, Karsten \& Bals, 1991) feststellen, sind mit ihrer geringen Bezahlung und schmalen gesellschaftlichen Reputation maximal weiblich dominiert, während die darauf bezogene, höher bezahlte und besser beleumundete Forschung zumindest partiell auch ein Arbeitsfeld für Männer darstellt - immerhin stammen knapp 30 Prozent der Beiträge der Frühen Bildung von Männern.

Eine weitere Frage ist, ob die Autor_innen der Zeitschrift an einer Hochschule oder in der pädagogischen Praxis tätig sind. Gerade in älteren Jahrgängen pädagogischer Periodika finden sich neben Beiträgen von Professor_innen und jüngeren Forscher_innen immer wieder Aufsätze von Praktiker_innen der pädagogischen und psychologischen Berufe. Dieses Phänomen wird in den letzten Jahren seltener; selbst in Zeitschriften mit starkem Praxisbezug schreiben inzwischen kaum noch Personen aus der Praxis, sondern überwiegend Wissenschaftler_innen. Die Frühe Bildung macht hier keine Ausnahme: Knapp 80 Prozent der Beiträge stammen von Autor_innen, die an Universitäten und Hochschulen beschäftigt sind, lediglich drei Prozent fallen in die Kategorie „Andere“. Auffällig ist, dass ein markanter Anteil der Autor_innen der Frühen Bildung an einem außeruniversitären Forschungszentrum beschäftigt ist (vgl. Abb. 3). Ohne es im Detail belegen zu können, würden wir als Ursache vermuten, dass insbesondere die Frühpädagogik seit ihrer ersten Boomphase - nach dem Jahr 1965 kaum an Hochschulen verankert war, sondern häufig an außeruniversitären Einrichtungen wie dem Deutschen Ju-

\section{Disziplin}

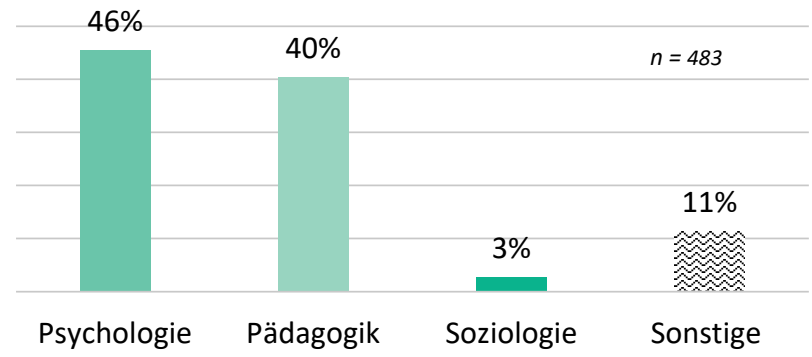

Abbildung 4. Autor_innen nach disziplinärem Hintergrund, prozentual.

gendinstitut oder dem Staatsinstitut für Frühpädagogik behandelt wurde, welche in der Forschungslandschaft seitdem eine erhebliche Bedeutung haben.

Die internationale Ausrichtung einer wissenschaftlichen Zeitschrift lässt sich anhand zahlreicher Parameter abschätzen. Ein relevanter Faktor ist sicherlich, in welchem Land die Autor_innen ihrem Beruf nachgehen. Für die Frühe Bildung zeigt sich, dass eine übergroße Mehrheit von 92 Prozent der Autor_innen einen Dienstort in Deutschland aufweist; lediglich sechs Prozent sind in der Schweiz beschäftigt, ein Prozent in Österreich sowie ein Prozent in weiteren, nicht-deutschsprachigen Staaten (vgl. Abb. 3). ${ }^{2}$ Damit unterscheidet sich die Frühe Bildung ein wenig von der Zeitschrift für Pädagogik, bei der 84 Prozent der Autor_innen an deutschen Institutionen beschäftigt waren (Zierer et al., 2013). Obwohl andere Indikatoren wie die akademische Mobilität oder die Nationalitäten der Autor_innen bei dieser Betrachtung unberücksichtigt bleiben, wird der Schwerpunkt der Frühen Bildung auf Forschungen aus Deutschland erkennbar.

Bei einem Zeitschriftenprojekt, das die Interdisziplinarität im Untertitel trägt, stellt sich die Frage, ob darin dennoch eine bestimmte Wissenschaftsrichtung Dominanz erreicht. Unsere Autor_innenanalyse deutet darauf hin, dass die Frühe Bildung ihr selbstgestecktes Ziel erreicht. Bei gut 200 Autor_innen (46 Prozent) ließ sich eine disziplinäre Zugehörigkeit zur Psychologie ermitteln, bei knapp 200 zur Pädagogik (40 Prozent). Soziologische Autor_innen waren mit einem Anteil von drei Prozent eher selten beteiligt (vgl. Abb. 4). Unter den 55 Autor_innen mit "sonstiger" Disziplin findet sich eine Vielzahl an Linguisten, was sich darauf zurückführen lässt, dass zahlreiche Beiträge den kindlichen Kompetenzerwerb im Bereich der Sprache fokussieren. Auffällig ist zudem, dass die Pädiatrie kaum vertreten ist: Die kinderärztliche Disziplin, die in den 1980er- und 1990er-Jahren beim Thema der frühen Kindheit noch diskursprägend

Bei fehlender Angabe eines Dienstortes $(n=10)$ in den Kurzinformationen zu den Autor_innen erfolgte die Kategorisierung nach Mailadresse. 
Disziplin, nach Zeitintervallen

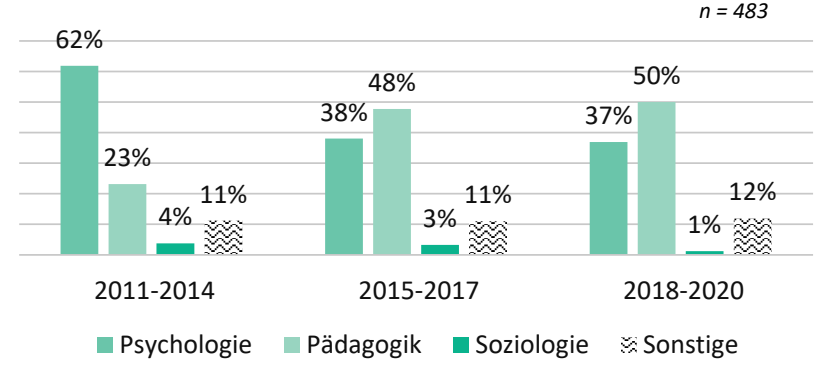

Abbildung 5. Autor_innen nach disziplinärem Hintergrund und Erscheinungszeit raum, nach Zeitintervallen, prozentual.

war (Berth, 2018), tritt in der Frühen Bildung fast nicht mehr auf.

Veränderungen werden deutlich, wenn man die zehn Jahrgänge in drei Perioden unterteilt und diese separat betrachtet. ${ }^{3}$ Demnach dominierten in der ersten Phase der Frühen Bildung Autor_innen mit psychologischer Herkunftsdisziplin; Pädagog_innen blieben deutlich in der Minderheit (vgl. Abb. 5). In den Phasen ab 2015 hingegen verschob sich dies: Bei leichter Dominanz der pädagogischen Autor_innen war das Kräfteverhältnis - wenn man diesen etwas martialischen Begriff verwenden mag - zwischen Psychologie und Pädagogik nun ungefähr ausgeglichen. Wir vermuten, dass dies mit Wechseln im Herausgebergremium zusammenhing, bei denen nach und nach einige der deutlich psychologisch orientierten Gründungsherausgeber abgelöst wurden durch neue Herausgeber, die stärker der Pädagogik zuzuordnen sind.

\section{Methodenanalyse}

Die Auswertung der verwendeten Methoden zeigt, dass es sich bei den Artikeln mehrheitlich um quantitative Forschungsbeiträge (59 Prozent) handelte. Dies spiegelt wohl auch die große Zahl der Autor_innen aus der Psychologie wider. Qualitative Designs - stärker verbreitet in den Erziehungswissenschaften und der Soziologie - waren in der Frühen Bildung mit 21 Prozent erheblich seltener. Im Vergleich dazu überraschte uns die Häufigkeit von MixedMethods-Ansätzen: Ziemlich genau jeder siebte Forschungsbeitrag arbeitete mit einer Quali-Quanti-Methodenkombination. Ohne dies belegen zu können, würden wir vermuten, dass diese Modernisierung der Methoden in wenigen etablierten Zeitschriften aus Psychologie und Pädagogik so weit fortgeschritten ist. Systematische Übersichtsarbeiten, unsere vierte Methodenkategorie, waren

\section{Methoden}

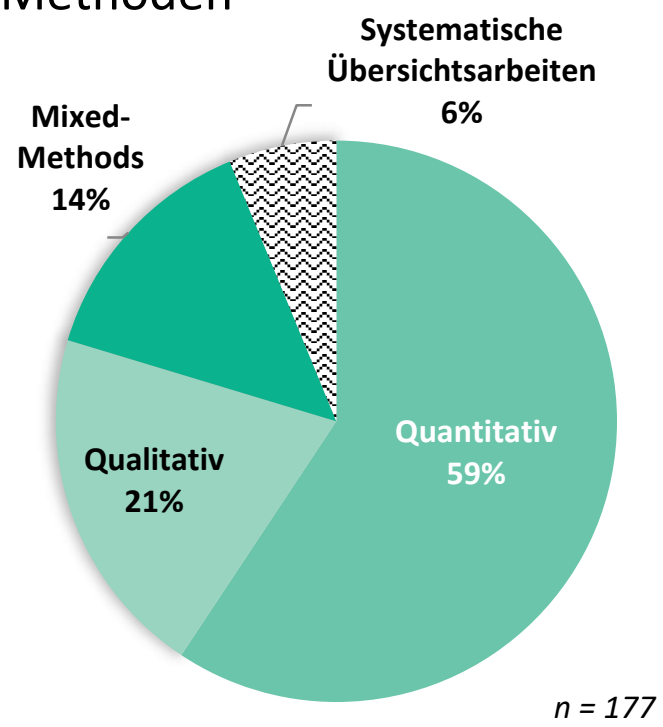

Abbildung 6. Beiträge nach Forschungsmethoden, prozentual.

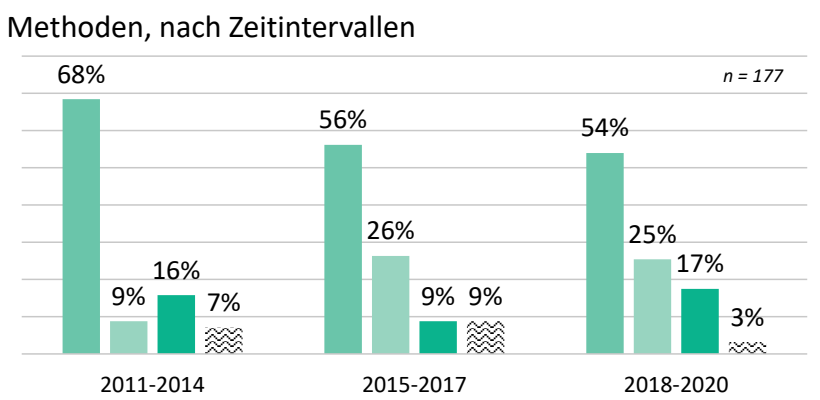

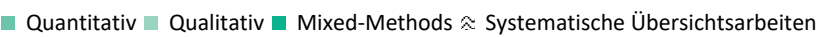

Abbildung 7. Beiträge nach Forschungsmethoden und Erscheinungszeitraum, prozentual.

mit einem Anteil von sechs Prozent vergleichsweise selten (vgl. Abb. 6).

Wiederum interessant ist die Entwicklung der Methoden im Zeitverlauf. Hier zeigt sich ein leicht sinkender Anteil der quantitativen Designs, der mit der schwindenden Dominanz der Psychologie in Zusammenhang stehen dürfte. Qualitative Methoden, in der Anfangszeit der Zeitschrift kaum vertreten, machten in den Jahren nach 2015 immerhin ein Viertel der Beiträge aus; auch das passt zum Befund einer wachsenden Präsenz der pädagogischen Disziplin. Bei den Mixed-Methods-Beiträgen ist die Entwicklung etwas uneinheitlich, in der Tendenz der letzten Jahre ist hier ebenfalls eine Zunahme erkennbar (vgl. Abb. 7). 


\section{Themenanalyse}

Womit beschäftigte sich die Frühe Bildung inhaltlich? Von den vier durch unsere Oberkategorien erfassten Hauptthemen - Kinder, Eltern, Personal und Organisation - dominierten zwei sehr deutlich: 44 Prozent der Beiträge legten ihren Fokus auf „Kinder“, 43 Prozent auf das Personal der Kindertagesbetreuung. Damit hatten fast neun von zehn der untersuchten 177 Forschungsarbeiten ihren Schwerpunkt in diesen Bereichen; die beiden anderen Oberkategorien „Eltern“ und „Organisation“ waren vergleichsweise selten vertreten (vgl. Abb. 8).

Aus unserer Sicht wäre das Gegenteil eine Überraschung: Würde sich eine Zeitschrift wie die Frühe Bildung nicht zentral mit Kindern beschäftigen, trüge sie das Adjektiv „früh“ wohl zu Unrecht im Titel. Gleichwohl kann man diesen Befund auch als Antwort auf das in der Kindheitssoziologie beliebte Argument lesen, über Kindheit werde in unserer Gesellschaft „nicht auf der Basis einer einfühlsamen Beobachtung der Kinder und ihres Wohlbefindens" gesprochen und entschieden (Bühler-Niederberger, 2020, S.24). Ohne dass wir beurteilen könnten, wie einfühlsam die 511 Autor_innen bei ihren 177 Beiträgen vorgingen, würden wir ihren thematischen Fokus zumindest als Interesse an Kindern deuten. (Bei einigen lag der Schwerpunkt sogar auf der Analyse von kindlichen Sichtweisen und Perspektiven; dazu unten mehr.)

Auch das zweite Hauptthema „Personal“ erstaunt wenig. Die Frühe Bildung fügte sich damit in einen wachsenden wissenschaftlichen wie auch gesamtgesellschaftlichen Diskurs über Kindertageseinrichtungen ein, der ungefähr mit der Veröffentlichung der ersten PISA-Befun-

\section{Hauptthemen}

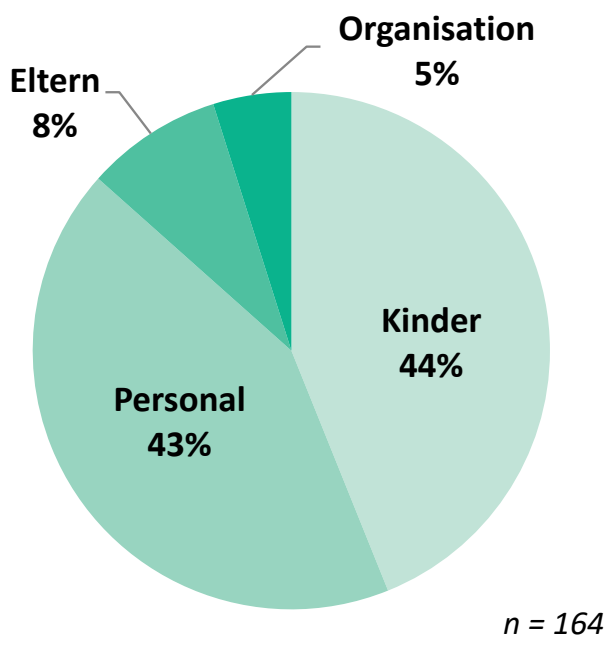

Abbildung 8. Beiträge nach Hauptthemen, prozentual. de im Jahr 2001 begann. Mit der in Deutschland allmählich wachsenden Überzeugung von der Kita als biografisch erstem Bildungsort wurde zunehmend die Frage gestellt, welche Beiträge das Kita-Personal hier potenziell leisten könne und faktisch leiste (Rauschenbach \& Berth, 2014). Die Frühe Bildung, könnte man sagen, versuchte hier Antworten zu geben.

Die beiden anderen Hauptthemen, „Organisation“ und „Eltern" wurden davon in den Hintergrund gedrängt, wobei wir vermutet hatten, dass gerade Eltern-Themen dank eines intensiveren wissenschaftlichen Diskurses über Familien als Bildungsort (Grgic \& Rauschenbach, 2021) in den letzten drei Jahren häufiger geworden wären. Das allerdings wird durch die Daten widerlegt: Die Frühe Bildung widmet sich dem Thema „Eltern“ von Anfang an mit kontinuierlicher, wenn auch geringer Intensität.

Versucht man die Themenwahl etwas detaillierter zu betrachten, bieten sich die Oberkategorien „Kinder" und „Personal“ wegen ihrer quantitativen Dominanz an. Beim Hauptthema „Kinder“ zeigt sich ein Fokus auf kindlichem Kompetenzerwerb: Mehr als die Hälfte der Beiträge mit Kinder-Schwerpunkt (das entspricht sogar 24 Prozent aller Beiträge der Zeitschrift) stellte Bildungsprozesse und kindliches Lernen ins Zentrum (vgl. Abb. 9). Dies lässt sich mit dem psychologischen Themenstrang der Zeitschrift in Verbindung bringen, der sich für die Entwicklung und Befähigung der Kinder interessiert. Sofern man die Skepsis gegenüber „sozialinvestiven Politikstrategien“ teilt, kann man darin auch Forschungen sehen, denen es weniger um Kinder in der Gegenwart geht, sondern vielmehr um ihre Zukunft als (mehr oder weniger) kompetente Arbeitnehmer (Klinkhammer, 2010).

Mit 15 Prozent deutlich seltener wird in Beiträgen mit Kinder-Fokus nach deren personalen und sozialen Charakteristika gefragt. Dies geschah meist in eher soziologisch orientierten Aufsätzen, deren Designs abhängige von unabhängigen Variablen unterschieden und beispielsweise untersuchten, welche Rolle Geschlecht oder Migrationshintergrund beim Aufwachsen von Kindern spielten. Eine Minderheit der Beiträge (5 Prozent) schließlich stellte dezidiert Kinderperspektiven in den Mittelpunkt. Wir würden das als Versuche interpretieren, die „Agency“ von Kindern nicht nur zu fordern, sondern tatsächlich zu erforschen, was in der Praxis oft schwieriger ist als programmatisch gefordert.

Beim zweiten Hauptthema der Zeitschrift, dem Personal, widersprach das Ergebnis unseren Vermutungen. Angenommen hatten wir, dass sich eine wissenschaftliche Zeitschrift mit dem Thema der frühen Bildung in hohem Maß mit der Akademisierung des entsprechenden Berufsfeldes beschäftigen würde. Das wäre inhaltlich u. a. zu begründen mit dem im Jahr 2004 begonnenen und zunächst sehr schnellen Ausbau hochschulischer Kindheitspädago- 
Hauptthema Kinder

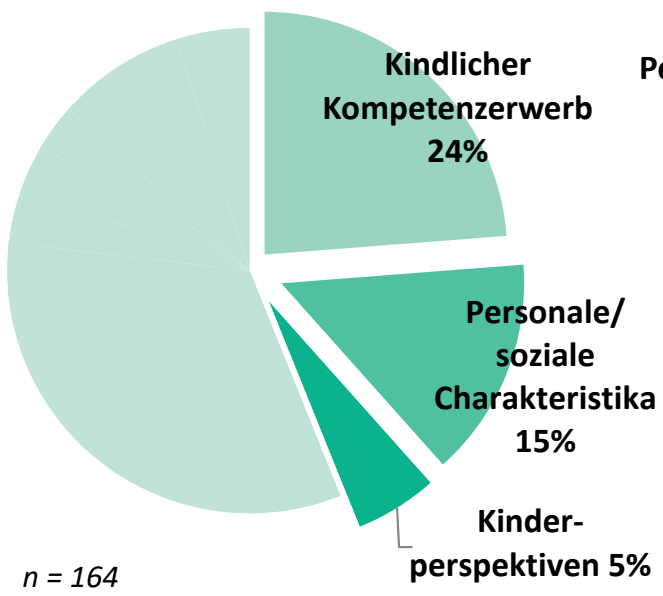

Hauptthema Personal

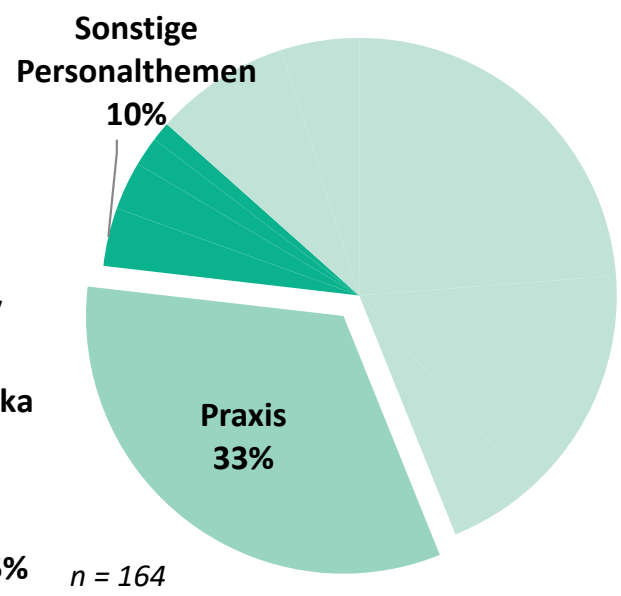

Abbildung 9. Beiträge mit Hauptthema Kinder, nach Unterthemen, prozentual / Beiträge mit Hauptthema Personal, nach Unterthemen, prozentual.

gik-Studiengänge in Deutschland (Autorengruppe Fachkräftebarometer, 2019); es entspräche auch der akademischen Gepflogenheit, insbesondere die eigene Berufs- und Argumentationswelt zu beobachten.

Die Frühe Bildung allerdings legte ihren Fokus bei Personalthemen eindeutig auf die Praxis: 33 Prozent aller Beiträge thematisierten z.B. pädagogische Orientierungen oder Kompetenzen des Praxispersonals. Weitere Aspekte, wie wir sie in unseren Unterkategorien erfasst hatten, wurden dahingegen sehr selten behandelt (vgl. Abb. 9). Immerhin, die Seltenheit der Forschungen zum „Ausbildungsort Fachschule" entsprach dann doch unserer Erwartung: Jene pädagogische Institution, an der auch heute noch die allermeisten Kita-Fachkräfte ausgebildet werden, wird auch in der Frühen Bildung kaum thematisiert. Hier zeigt sich wohl wieder einmal das "deutsche Schisma" (Baethge, 2007) einer strikten Trennung von hochschulischem und berufsschulischem System, das zu einer wechselseitigen Nicht-Wahrnehmung führt.

\section{Ein Resümee}

Ein Versuch, diese Inhaltsanalyse in wenigen Sätzen zusammenzufassen, führt zu folgender Bilanz: Die Autor_innen der Frühen Bildung sind überwiegend weiblich, verfassen ihre Beiträge meist in Teams und arbeiten größtenteils an Universitäten und Hochschulen, teilweise an außeruniversitären Forschungsinstituten. Entsprechend gilt im Umkehrschluss: Männer als Autoren sind selten, Einzelautor_innenschaften noch seltener, Praktiker_innen schreiben in dieser Zeitschrift so gut wie gar nicht. Zum Autor_innenstamm gehören weit überwiegend
Psycholog_innen und Pädagog_innen, wobei erstere ihre anfängliche Dominanz im Lauf der Jahre abgegeben haben, womit das „Kräfteverhältnis“ zwischen beiden Disziplinen inzwischen ausgeglichen ist. Mit dieser Annäherung ging auch die zu Beginn ausgeprägte quantitative Ausrichtung der Zeitschrift etwas zurück; inzwischen sind qualitative Methoden häufiger präsent, und auch MixedMethods-Ansätze erreichen einen markanten Anteil. Naheliegenderweise konzentriert sich die Frühe Bildung auf zwei Hauptthemen, die Kinder sowie das Kita-Personal; Organisations- und Elternthemen sind daneben vergleichsweise randständig. In den Forschungsarbeiten über Kinder steht deren Kompetenzerwerb im Vordergrund; eigenständige Kinderperspektiven werden deutlich seltener behandelt. Beiträge, die sich mit Personalfragen beschäftigen, thematisieren überwiegend die Kita-Praxis; demgegenüber sind Ausbildungsorte wie Hochschulen oder Fachschulen im Themenspektrum der Zeitschrift wenig vertreten.

Zwei Limitierungen seien am Schluss noch erwähnt. Erstens haben wir nur Beiträge aus den beiden Forschungs-Rubriken der Zeitschrift untersucht; in den anderen Teilen (Rezensionen, Diskussionen, Innovationen sowie Informationen) fänden sich möglicherweise andere inhaltliche Schwerpunkte sowie eine anders strukturierte $\mathrm{Au}-$ tor_innenschaft. Zweitens - und das ist die wichtigere Begrenzung - bringt die Zuordnung aller Texte zu einer inhaltsanalytischen Kategorie mit sich, dass jeder Beitrag nur mit dem von uns als zentral betrachteten Aspekt in die Auswertung eingeht. Das bedeutet, dass alle weiteren thematischen Facetten durch unser Verfahren ausgeblendet werden. Dieser Nachteil wird besonders bei komplexeren Texten - wie sie in der Wissenschaft nun einmal häufig sind - relevant; gleichwohl steht dem der Vorteil gegen- 
über, dass das Verfahren quantifizierend ist und überprüfbare Ergebnisse erbringt. Auch deshalb schien es uns passend zu sein für eine Zeitschrift, die die Empirie so wertschätzt wie die Frühe Bildung.

\section{Literatur}

Autorengruppe Fachkräftebarometer (2019). Fachkräftebarometer Frühe Bildung. München: DJI/WiFF.

Baethge, M. (2007). Das deutsche Bildungs-Schisma: Welche Probleme ein vorindustrielles Bildungswesen in einer nachindustriellen Gesellschaft hat. In D. Lemmermöhle \& M. Hasselhorn (Hrsg.), Bildung und Lernen. Humanistische Ideale, gesellschaftliche Notwendigkeiten, wissenschaftliche Erkenntnisse (S.93-116). Göttingen: Wallstein.

Becker-Stoll, F., Fröhlich-Gildhoff, K., Hasselhorn, M., NentwigGesemann, I., Petermann, F., Roßbach, H.-G., Schneider, W. \& Viernickel, S. (2011). Editorial. Frühe Bildung, 0 (1), 1. https://doi. org/10.1026/2191-9186/a000006

Berth, F. (2018). Der skeptische Blick. Pädiatrische Perspektiven auf frühe institutionelle Kinderbetreuung in der Bundesrepublik. Zeitschrift für Soziologie der Erziehung und Sozialisation, 38 (1), 4-23.

Bray, N. \& Major, C. (2011). Status of Journals in the Field of Higher Education. The Journal of Higher Education, 82 (A), 479-503. https://doi.org/10.1353/jhe.2011.0020

Bühler-Niederberger, D. (2020). Warum es Kindheit nicht gibt. Eine skeptische Geschichte des Aufwachsens. Kursbuch, 56 (201), 24- 40 .

Fleck, L. (1936/1982). Erfahrung und Tatsache. Gesammelte Aufsätze. Frankfurt/Main: Suhrkamp.

Grgic, M. \& Rauschenbach, T. (2021). Bildungsort Familie. Informelle Bildung. In J. Ecarius \& A. Schierbaum (Hrsg.), Handbuch Familie. Erziehung, Bildung und pädagogische Arbeitsfelder. Wiesbaden: Springer VS.

Kauder, P. \& Vogel, P. (Hrsg.) (2015). Lehrbücher der Erziehungswissenschaft - ein Spiegel der Disziplin. Bad Heilbrunn: Klinkhardt.
Klinkhammer, N. (2010). Frühkindliche Bildung und Betreuung im „Sozialinvestitionsstaat“ - mehr Chancengleichheit durch investive Politikstrategien? In D. Bühler-Niederberger, J. Mierendorff \& A. Lange (Hrsg.), Kindheit zwischen fürsorglichem Zugriff und gesellschaftlicher Teilhabe (S.205-228). Wiesbaden:VS.

Martini, R. \& Dees, W. (2012). Qualifizierungsschriften in der Bildungsforschung 1998 bis 2007. In H. Weishaupt \& M. Rittberger (Hrsg.), Bildungsforschung in Deutschland - eine Situationsanalyse (S. 43 -64). Frankfurt/Main: DIPF.

Rabe-Kleberg, U., Krüger, H., Karsten, M. E. \& Bals, T. (1991). Dienstleistungsberufe in Krankenpflege, Altenpflege und Kindererziehung: Pro Person. Bielefeld: KT.

Rauschenbach, T. \& Berth, F. (2014). Kindergärtnerin - Erzieherin - und dann? Frühpädagogische Professionalisierung zwischen Stagnation und nachholender Modernisierung. Frühe Bildung, 3 (4), 238 - 242. https://doi.org/10.1026/2191-9186/a000179

Tenorth, H.-E. (1986). Transformationen der Pädagogik - 25 Jahre Erziehungswissenschaft in der „Zeitschrift für Pädagogik“. Zeitschrift für Pädagogik, 20. Beiheft, $21-85$.

Zierer, K. Ertl, H., Phillips, D. \& Tippelt, R. (2013). Das Publikationsaufkommen der Zeitschrift für Pädagogik im deutsch-englischen Vergleich. Zeitschrift für Pädagogik, 59 (3), 400 - 424.

\section{Autorenschaften}

Die Mitautorin ist erreichbar unter: Hanna Schneider, hanna. schneider1995@web.de

\section{Förderung}

Der Beitrag entstand im Rahmen des von der DFG geförderten Projekts „Von der Nothilfe zur Bildung“ (BE 6913/1), das von Felix Berth geleitet wird und bei dem Hanna Schneider Anfang 2021 ein Forschungspraktikum im Rahmen ihres Masterstudiums der Early Childhood Studies (Pädagogische Hochschule Weingarten / Pädagogische Hochschule St. Gallen) absolvierte.

\section{Felix Berth}

Abteilung Kinder und Kinderbetreuung

Deutsches Jugendinstitut

Nockherstr. 2

81541 München

berth@dji.de 